Epinoches, les a jetćes dans la rigole voisine et là, peu à peu, elles se sont multipliées; les nombreux canaux dasséchement et de. drainage de la vallée du Rhône, en Valais, se sont graducllement infestés d'Epinoche, qui en appréciait les eaux lentes el chaudes et, par étapès, Ia tache primitive s'est étendue vers l'aval.

: Ultérieurement, on signale la présence de l'Epinoche sur la côte vaudoise, vers Villeneuve. En Mars 1928, on en pêche un individu devant. Thonon. Depuis cette date, il n’a plus été signalí de capture. Les eaux froides du Léman, l'absence de végétation sur les grèves sont heureusement des conditions défavorables à sa propagation. Par une faveur de la nature, le Léman semble devoir rester à l'abri de l'Fpinoche, sauf quel.ques rares témoins.

En l'espèce, la nature a bien fait les choses ct l'équilibre biologique du Léman, détruit par l'Homme il y a trente aus et que de pénibles eflorts tendent aujourd'hui à resiaurer, n'a pas cu à souffrir de l'introduction de ce dangereux intrus.

\title{
EXPLOITATION DES ÉTANGS
}

\author{
Par M. Phmmbert PANNETIER \\ Secrétaire général de l'Union nationale des Pêcheurs aux filets.
}

(Suite) (1)

Disposilif de pêcherie le pius recommandable pour la pêche des alevins.

En ce qui concerne le disposilif de pêchcric des élangs d'empoisson. rage, le plus recommandable, quand il y a possibilité de l'établir, est certainement celui employé couramment dans la Dombes, pour les petits comme pour les grands fonds. Il consiste en une pêcherie en avant et à proximité de la bonde, proportionnée en grandeur à celle de l'étang et permettant la capture, facile et rapide, de toute la pêche au moyen d'un filet.

Les autres, usilés dans certaines régions, sont l's suivants :

.' Un bick en avant de da bonde permellaul l'coulement de l'ear et to rassemblement du Poisson.

Ce procédé a le sros inconvénient de réunir le Poisson parlois sur une grande longueur el de mottre los pêcheurs dans l'obligation de le tainer dans la vase pour le capturer, e gui n'est suère linvorable à la conserva tion de n’importe quel Poisson, mais surtoul des alevins.

Si ce bief était entreteuu, c'est-ì-dire de temps à antre débarmassé de

(1) Voir Bulletin, Miti iģo, p. 259 . 
ia vase que l'eau y accumule, il n'y aurait que demi-mal, mais, malhoureusement, actuellement la main-d'œuvre lait de plus en plus défaut pour accomplir un travail bien plus important que celui consistant à meltre en état une pêcherie.

$2^{\circ}$ Une pêcherie, désignée couramment sous le nom de " panier ", située à l'arrière de la bonde et construite généralenent en maçonnerie.

Ce procédé est usité surtout dans les régions où les étangs ne sont jamais mis en culture, pas même à sec de temps à autre pour assainir le terrain. L'abondance de la vase est telle en avant de la bonde qu'il y aurait impossibilité matérielle d'y récupérer le Poisson. Aussi, que voit cn à la pêche ? Cette masse de vase passer, à chaque coup de vanne, à l'arrière dans la pêcherie, mélangée avec le Poisson et toutes sorles de détritus provenant de l'étang.

Après avoir été roulés et retournés par les tourbillons dans le canal et la pêcherie en maçonnerie, nombreux sont les gros Poissons auxquels il manque passablement d'écailles et qui, sous peu, périront malgré tous les soins possibles et imaginables.

Quant aux petits, que de viclimes immédiatement el combien de pertes, par la suite, ce qui explique surabondamment le déchet constaté aux pêches résultant de ces empoissonnages.

Sans contestation possible, les pêcheries situées en avant de la bonde sont les plus recommandables pour toutes les pêches d'étangs, mais sur lout pour les pêches d'empoissonnage, à condition, toutefois, qu'elles soient assez profondes et mises en état tous les deux ou trois ans, pour permettre la capture du Poisson en cau abondante et propre.

\section{Choix des géniteurs.}

Carpes de pose. - La Carpe étant l'élément le plus important de la I roduction des étangs, je commencerai par elle.

Le choix des Carpes de pose, comme nous les désignons vulgairemerit et couramment, est, à l'origine, une précaution essentielle pour réaliser prar la suite de fortes récoltes en Poisson de qualité.

Le mieux, certainement, serait de s’adresser, pour avoir des Carpes de pose d'origine sélectionnée, aux éminents pisciculteurs qui ont mis en ceuvre toute leur science et leur bonne volonté pour régénérer la Carpo commune française (de crainte d'oublier peut-ètre le plus méritant, je ne Ies désignerai pas par leur nom). Mais, peu nombreux sont les propriétaires d'étangs, susceptibles de faire de l'empoissonnage, qui se trouvent à portée de ces fournisseurs éventucls. D'autre part, le transport par chemin de fer est très délicat, surtout par petite quantité.

Il y a donc lieu, notamment, pour ceux qui ne peuvent se payer le luxe de s'approvisionner auprès de ces personnalités marquantes, de chercher. par leurs propres moyens, une solution susceptible de leur donner satisfaction. 
Pour cela, avec un peu d'esprit de suite et de la persévérance, en suivant mes conseils, ils y arriveront.

En principe, il faut, pour avoir une progéniture de qualité, choisir (ies pères et mères sains, ayant acquis, en trois ou quatre ans pour des Carpes communes et cinq ou six ans, même plus, pour des Carpes "cuir " ou de Galicie, une grossetir maxima.

Par grosseur maxima, il faut entendre Carpes ayant atteint : - de 3 à 4 livres à trois ans, 5 à 6 livres à quatre ans d'existence pour les Carpes communes; - et de 7 à 8 livres à cinq ans et 9 à 10 livres à six ans d'exis tence s'il s'agit de Carpes cuir ou de Galicie.

En un mot, choisir toujours les plus belles de la promotion, soit dans sa récolte ou celle de son voisin s'il y a lieu, en admettant même qu'un nombre important de kilomètres soit à faire pour se les procurer.

Par ce moyen et, naturellement, en évitant la surcharge des étangs, source de dégénérescence des meilleurs produits, on arrivera, sans aucun doute, à améliorer dans une certaine mesure notre production de Carpes.

Abandonnons la théorie de certains propriétaires d'étangs qui recher chent comme géniteurs des sujets d'une livre, dont il est impossible de déterminer l'âge et maigres comme des clous, parce qu'ils posent plus sûrement : recherchons la qualité avant la quantité !

Tanches de pose. - En ce qui concerne la Tanche, nos hommes de science, nos pisciculteurs expérimentés, s’ils en parlent, ne semblent pas très préoccupés de son avenir.

Pourtant, le champ des investigations à son sujet est bien aussi vaste sinon plus, que pour la Carpe.

$\mathrm{Si}$, dans une certaine région, la maladie de la Tanche (le ver) est en régression, d'autre part, la zone infestée ne s'élargit-elle pas de plus en plus chaque année ? Ceci signifie, malheureusement, que cette maladis n'est pas encore sur le point de disparaître!

Sans être grave, la question doil néanmoins retenir l'attention de tous ceux qui s’intéressent à la pisciculture.

N'y aurait-il pas lieu de rechercher les causes de la régression cons. tatée et d'en tirer les conclusions nécessaires à la lulte qui doit être tentấe en vue de la disparition du Ver?

Celui-ci disparu, une grosse augmentation de production en résulterait eí, d'autre part, une facilité de vente inestimable.

En attendant cette éventualité, choisissons toujours, pour faire des ale. vins, dits "éguillons", et, plus tard, des Tanchons de qualité, des Tanches bicn saines de quatre années et pesant au minimum 400 grammes.

\footnotetext{
(A suivre).
} 